\title{
The usefulness of 18F-FDG PET/CT in follow-up and recurrence detection for patients with lung carcinoma and its impact on the survival outcome
}

\author{
Gihan Hassan Gamal(D
}

\begin{abstract}
Background: We assess the usefulness of 18F-FDG PET/CT for detection of recurrent or residual tumor in post treatment patients with NSCLC and comparing the results with CECT, and we evaluate its impact to the clinical assessment and overall survival of lung cancer patients.

Results: A prospective study of 63 patients with potentially resectable NSCLC, were divided into 2 groups according to the initial staging of the disease and the early response to treatment.

Group A ( $n=29)$ patients were treated by curative treatment, and group B $(n=34)$ patients were treated by palliative treatment.

Evaluation of patients was done during the follow-up period clinically every 3 months and by 18F-FDG PET/CT and CECT imaging at 6 months intervals.

In group A, 18F-FDG PET/CT correctly diagnosed all recurrent or residual tumors $(n=7)$ whereas CECT diagnosed only 5 with 2 false negative cases.

In group B, 18F-FDG PET/CT correctly diagnosed all recurrent or residual tumors ( $n=23$ ) whereas CECT diagnosed 16 patients with 7 false negative cases.

By comparison of 18F-FDG PET/CT and CECT in detection of residual or recurrent lung cancer $(n=30)$, the sensitivity, specificity, PPV, NPV, and accuracy of 18F-FDG PET/CT were 100\%, 92\%, 92\%, 100\%, and 96\% respectively, while of CECT were $72 \%, 95 \%, 94 \%, 79 \%$, and $84 \%$ respectively in correlation with reference standard data. The calculated SUV max ranged from 2.1 to 4.9 .

There was a significant difference in overall survival between patients in routine scan who had positive 18F FDG PET/ $C T$ result (median survival 18 months) and those who had negative result (median survival 45 months) $(P<0.0001)$.

Conclusion: 18 F-FDG PET/CT plays an important role in distinguishing post treatment changes from tumor recurrence in patients with lung cancer. Follow-up or surveillance 18 F-FDG PET/CT is a prognostic indicator for overall survival of patients.
\end{abstract}

Keywords: Non-small cell lung cancer (NSCLC), 18F-FDG PET/CT, Overall survival

Correspondence: dr.gh_006@yahoo.com

Radiodiagnosis Department, October 6 University, Giza, Egypt

๑) The Author(s). 2021 Open Access This article is licensed under a Creative Commons Attribution 4.0 International License, which permits use, sharing, adaptation, distribution and reproduction in any medium or format, as long as you give appropriate credit to the original author(s) and the source, provide a link to the Creative Commons licence, and indicate if changes were made. The images or other third party material in this article are included in the article's Creative Commons licence, unless indicated otherwise in a credit line to the material. If material is not included in the article's Creative Commons licence and your intended use is not permitted by statutory regulation or exceeds the permitted use, you will need to obtain permission directly from the copyright holder. To view a copy of this licence, visit http://creativecommons.org/licenses/by/4.0/. 


\section{Background}

Lung carcinoma is one of the leading causes of cancerrelated mortality worldwide. It is divided histologically into two main types: NSCLC accounts for $85 \%$ of cases and SCLC $10-15 \%$ of cases [1]. The overall 5-year survival rate for patients with lung cancer is $17.4 \%$ [2]. However, an important clinical problem in patients treated for lung cancer is the evaluation of treatment response and detection of recurrence. The post therapeutic anatomic and tissue alterations resulting from surgery and/or radiotherapy of lung cancer can make it difficult to discriminate necrotic tumor or fibrotic scar tissues from tumor relapse [3].

The follow-up radiological imaging by using response evaluation criteria in solid tumor (RECIST criteria) of lung cancer patients using CT with or without contrast media to detect tumor recurrence was widely accepted in determining treatment response. However, it depends on the size and mass changes occurred to the tumor post treatment, irrespective of the tumor viability, thus lowering its diagnostic performance with decreasing sensitivity and specificity for detection of cancer recurrence [4].

Although18F-FDG PET/CT may detect early functional changes occurred post treatment as well as structural changes, it is not included as routine in follow-up or surveillance after therapy [5].

The imaging principle of $18 \mathrm{~F}$ FDG PET/CT depends on the substantially different central metabolic pathways in malignant tissue. Thereby, most of the cancer cells show elevated levels of glucose uptake and thus produce high level of lactate compared to nonmalignant cells which may be detected by 18 F FD PET/CT. The FDG uptake level can be assessed by using standardized uptake value (SUV) [6].

The aim of this study was to assess the usefulness of 18F FDG PET/CT for detection of residual or recurrent tumors in post treatment NSCLC patients comparing the results with CECT, and to evaluate its impact to the clinical assessment and survival outcome of lung cancer patients.

\section{Methods}

\section{Selection of patient and their preparation}

This prospective study included 63 patients with potentially resectable NSCLC with age ranged from 49 to 75 years. Patients were examined at our radiology department over a period from February 2017 to February 2021.According to the initial staging of the tumor and the early response to treatment, all patients were divided into 2 groups: Group A, patients were treated with curative treatment and, group B patients were treated with palliative treatment.

18F FDG PET/CT and CECT were performed to all patients as requested.
Serum creatinine was asked to be below $2 \mathrm{mg} / \mathrm{dL}$ prior to the study and the blood glucose level $(<160 \mathrm{mg} / \mathrm{dL})$ was controlled before the date of imaging to avoid FDG misinterpretation.

The patients were fasted for at least $6 \mathrm{~h}$ prior to the scan. They were requested to rest in a quiet room and try to avoid vigorous movement and talking as minimal as possible to minimize physiological uptake of FDG.

Informed consent has been taken from all the patients as according to the ethical committee regulations.

\section{Technique}

18 F-FDG PET/CT is performed using PET/CT scanner (Discovery STE; GE Healthcare, Boston, USA). In total, $5.5 \mathrm{MBq} / \mathrm{kg} 18 \mathrm{~F}-\mathrm{FDG}$ dose was injected intravenously 60 min before starting the scan. A PET emission scan was performed over several bed positions from 5-7 for 2 min per bed position with axial field of view of $21.6 \mathrm{~cm}$ per bed position and in plane spatial resolution of $2 \mathrm{~mm}$ from the base of the skull down to the mid-thigh at 2 min per bed position.

The following diameters were used in performing the CECT, $120 \mathrm{kV}, 350 \mathrm{mAs}, 0.5 \mathrm{~s}$ tube rotation, slice thickness of $5 \mathrm{~mm}$, with $8 \mathrm{~mm}$ table feed, and $3 \mathrm{~mm}$ incremental reconstruction. Transaxial PET and CT images were reconstructed, fused, and reformed into coronal and sagittal images, and data were generated. The SUV$\max$ in the volume of interest was measured.

The 18F FDG PET/CT images were interpreted via two experienced radiology consultants with more than 5 years of experience with inter-observer agreement.

Two experienced radiology consultants, more than 5 years of experience, interpreted the CECT images with inter observer agreement.

The readers were blinded to each other and reports were compared. A comparison between 18F FDG PET/ $\mathrm{CT}$ and CECT findings was done and it was pathologically confirmed by biopsy, clinical evaluation, and subsequent imaging progression of patients.

\section{Images analysis \\ Visual analysis}

Any focus of elevated FDG metabolism, and not located in areas of normal FDG uptake, was considered positive.

\section{Quantitative analysis}

18 F-FDG PET/CT was measured by $\mathrm{SUV}_{\max }$, we manually placed the region of interest on each lesion of abnormal uptake then it was calculated.

\section{Statistical analysis}

Sensitivity, specificity, positive and negative predictive value (PPV and NPV), and accuracy of 18F FDG PET/ $\mathrm{CT}$ and CECT for detection of tumor recurrent and/or 
residual tumor were calculated and compared using McNemar $T$ test. $P$ value was set to be statistically significant when it is $\leq 0.05$.The $\mathrm{SUV}_{\max }$ of tumor was quantitatively and qualitatively evaluated. All the collected data were loaded and coded by using the Statistical Package for Social Sciences (SPSS version 22).

\section{Patient's overall survival measures}

The overall survival of the patients in routine scan was measured as the time interval between the follow-up $18 \mathrm{~F}$ FDG PET/CT scan and the end of data collection. The statistical analysis was done to determine the relationship between the follow-up 18F FDG PET/CT results and overall survival of patients.

\section{Results}

In this prospective study, we analyzed 63 patients with potentially resectable NSCLC with age ranged from 49 to 75 years. Patients were examined at our radiology department over a period from February 2017 to February 2021. The final staging of the disease was as follows: Stage I, 10 patients; stage II, 11 patients; stage IIIA, 18 patients; and stage IIIB, 24 patients. Serum creatinine was $(<2 \mathrm{mg} / \mathrm{dL})$ and the blood glucose level $(<160 \mathrm{mg} /$ $\mathrm{dL}$ ) before the imaging.

The therapeutic regimens ranged from surgery/and or radiotherapy and occasionally associated with chemotherapy (Table 1).

According to the initial staging of the tumor and the early response to treatment, all the patients were divided into 2 groups:

Group A $(n=29)$, in this group, patients were treated by curative treatment, 21 patients with stages I and II and 8 patients with stage IIIA. None of these patients showed a persistence intrathoracic radiological abnormality for 3 months after the end of treatment.

Group B $(n=34)$, in this group, patients were treated by palliative treatment. All of these patients showed persistence intrathoracic radiographic abnormality for 3 months after the end of treatment.

Table 1 Treatment regimen of groups A and B patients

\begin{tabular}{lll}
\hline & Treatment regimen & Patient number \\
\hline Group A $(n=29)$ & Surgery & 16 \\
& Surgery+radiotherapy & 6 \\
& Surgery +chemotherapy & 6 \\
& Chemotherapy +radiotherapy & 1 \\
Group B (n=34) & Radiotherapy & 16 \\
& Radiotherapy+chemotherapy & 14 \\
& Surgery +chemotherapy & 4 \\
\hline
\end{tabular}

Follow-up assessment was performed more than 6 months after the primary treatment completion. Each patient was clinically evaluated every 3 months during the treatment follow-up period. 18F FDG PET/CT and CECT imaging was performed at 6 months intervals for 6-36 months, however, if the patient presented by suspicious symptoms or if the radiological imaging suggested residual or recurrence of the tumor, a biopsy was obtained and clinical and radiological follow-up was performed.

\section{F FDG PET/CT and CECT imaging revealed}

In group $\mathrm{A}$, all recurrent tumors $(n=7)$ were correctly diagnosed by $18 \mathrm{~F}$ FDG PET/CT (high FDG uptake) whereas CECT correctly detected recurrent tumors in 5 patients with 2 false negative cases, as metastatic mediastinal lymphadenopathy were not detected by CT (Figs. 1 and 2).

18F FDG PET/CT and CECT correctly identified the regression of tumor in 21 of 22 patients. Detection of one false positive case by 18F FDG PET/CT imaging in a patient with radiation pneumonitis and one false positive case by CECT was noted (Table 2).

The sensitivity, specificity, PPV, NPV, and accuracy of 18F FDG PET/CT in detecting tumor recurrence in correlation with reference standard data were $100 \%$, $97 \%$, $93 \%, 100 \%$, and $98 \%$ respectively. The accuracy of CECT was $91 \%$.

In group $\mathrm{B}$, all recurrent or persistent cases $(n=23)$ of lung tumor revealed increase FDG uptake by 18F FDG PET/CT with no false negatives. CECT results revealed stable appearance with no regression of the tumor in 16 cases while 7 cases where false negative (Fig. 3).

Eleven patients showed absence of recurrent tumors for 15-36 months after treatment, they were correctly identified by 18F FDG PET/CT in 9 cases and by CECT in 10 cases.

In this group of patients, 18F FDG PET/CT showed two false positive cases $(1$ infectious process and 1 radiation pneumonitis) and CECT showed one case false positive as it showed tissue changes suspicious for tumor recurrence (Table 3).

The sensitivity, specificity, PPV, NPV, and accuracy of $\mathrm{PET} / \mathrm{CT}$ in detecting tumor recurrence in correlation with reference standard data were $100 \%, 81 \%, 92 \%, 100 \%$, and $94 \%$ respectively. The accuracy of CECT was $78 \%$.

By comparison of both groups for 18F FDG PET/ $\mathrm{CT}$ and CECT in detection of residual or recurrent lung carcinoma revealed, sensitivity, specificity, PPV, NPV, and accuracy of 18F FDG PET/CT were $100 \%, 92 \%, 92 \%, 100 \%$, and $96 \%$ respectively, while of CECT were $72 \%, 95 \%, 94 \%, 79 \%$, and $84 \%$ respectively in correlation with reference standard data. 


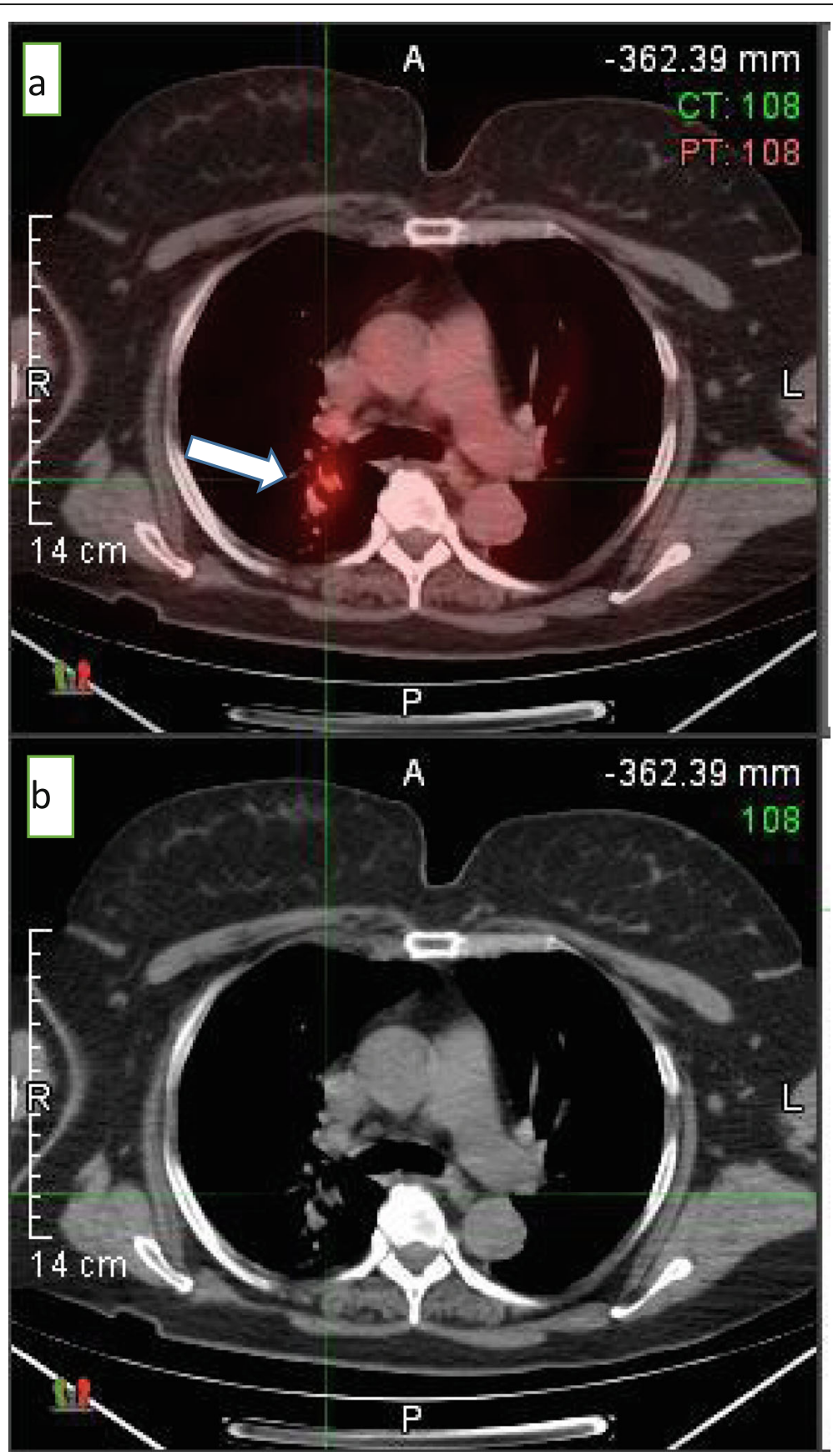

Fig. 1 (a-b) 56-year-old female with recurrent lung carcinoma 18F-FDG PET/CT was performed 1 year post treatment, revealed $3.2 \mathrm{~cm}$ AP $\times 2.7 \mathrm{~cm}$ wide FDG avid mass (SUV $\max 3.4$ ) in the posterior segment of right upper lung lobe (arrow). Right retrocaval pretracheal lymph node with lowgrade activity tumor bearing lymph node was noted (a). CT imaging revealed atelectasis and/or pneumonitis measuring $3.2 \mathrm{~cm} A P \times 2.7 \mathrm{~cm}$ wide at the posterior segment of right upper lung lobe with no metastatic lymphadenopathy detected (b)

The calculated SUV max ranged from 2.1 to 4.9 .

\section{The overall survival analysis}

The 18F FDG PET/CT scans revealed from 63 patients were grouped as negative or positive reports for tumor recurrence or metastasis.
18F FDG PET/CT scans for recurrence or metastasis display negative result in 33 patients and positive in 30 patients.

Of the negative 18F FDG PET/CT results, $40 \%$ were obtained 6-24 months after completion of treatment and $60 \%$ more than 24 months.

Of the positive scans, $45 \%$ were obtained 6-24 months after completion of treatment and 55\% more than 24 months. 

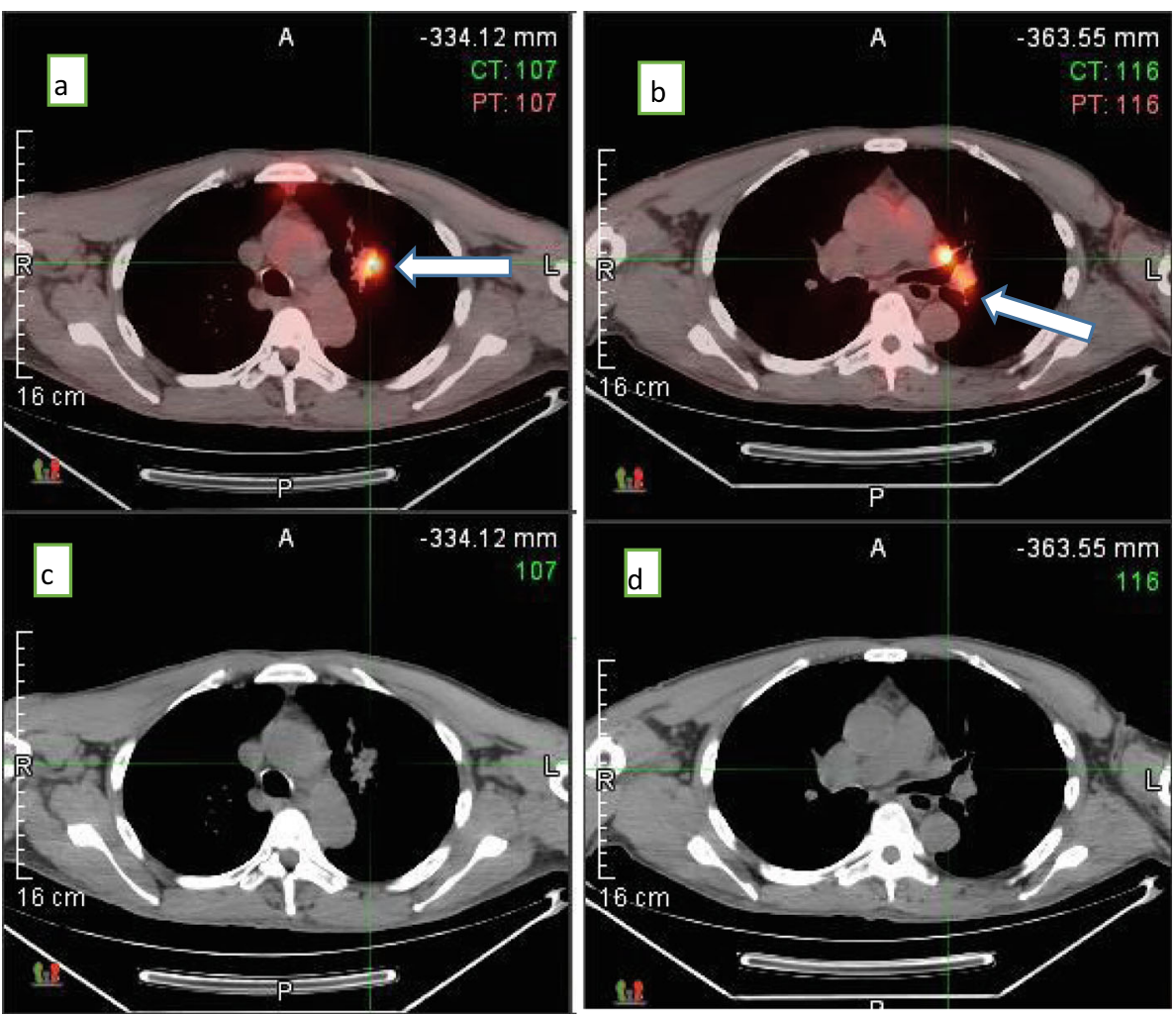

Fig. 2 (a-d) 72-year-old male with recurrent lung tumor post therapy 18F-FDG PET/CT revealed $3.6 \mathrm{~cm}$ AP $\times 2.5 \mathrm{~cm}$ wide medial left upper lobe $\left(S U V_{\max } 4.9\right)$ speculated mass with surrounding non FDG avid atelectasis (arrow). Contiguous left hilar metastatic lymphadenopathy (SUV $V_{\max }$ 4.9)

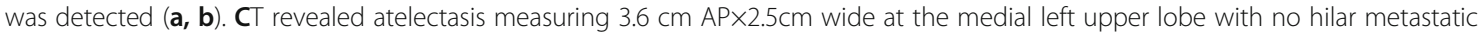

lymphadenopathy detected (c, d)

A significant difference with $P$ value $<0.0001$ has been revealed in overall survival by using the Kaplan-Meier analysis between the patients in routine scan who had positive 18F FDG PET/CT result (median survival 18 months) and those who had negative result (median survival 45 months) (Fig. 4).

\section{Discussion}

The early detection of disease recurrence and assessment of the therapeutic response in patients treated for lung cancer has been of great importance [7].

18 F-FDG PET/CT is a valuable routine imaging tool in the diagnosis and staging of lung cancer; however, its

Table 2 Group A ( $n=29)$ follow-up imaging results (correlation with final diagnosis)

\begin{tabular}{lll}
\hline & 18 F-FDG PET/CT & CECT \\
\hline Remission & 21 & 21 \\
True negative & 1 & 1 \\
False positive & & \\
Recurrent or residual & 0 & 2 \\
False negative & 7 & 5 \\
True positive & & \\
\hline
\end{tabular}

value in follow-up and surveillance is still controversial [8]. In this study, we assess the usefulness of 18 F-FDG $\mathrm{PET} / \mathrm{CT}$ in differentiating post treatment changes in patient with NSCLC from tumor relapse comparing the results with CECT, and we evaluate its impact to the clinical assessment and overall survival of lung cancer patients.

The present study found that18 F-FDG PET/CT was more accurate than CECT in distinguishing persistent or recurrent tumor from fibrotic scar tissue in patients treated for NSCLC. All of the recurrent tumors were correctly diagnosed by 18 F-FDG PET/CT, 7 patients in group A (patients were treated with curative treatment) and 23 in group B (patients were treated with palliative treatment). By comparison of 18 F-FDG PET/CT and CECT in detection of residual or recurrent lung cancer, the sensitivity, specificity, PPV, NPV, and accuracy of 18 F-FDG PET/CT were 100\%, 92\%, 92\%, 100\%, and 96\% respectively while those of CECT were $72 \%, 95 \%, 94 \%$, $79 \%$, and $84 \%$ respectively, in correlation with reference standard data. The calculated $\mathrm{SUV}_{\max }$ ranged from 2.1 to 4.9 .

These results were in concordance with a previous meta-analysis done by You J et al. who reported that 18 

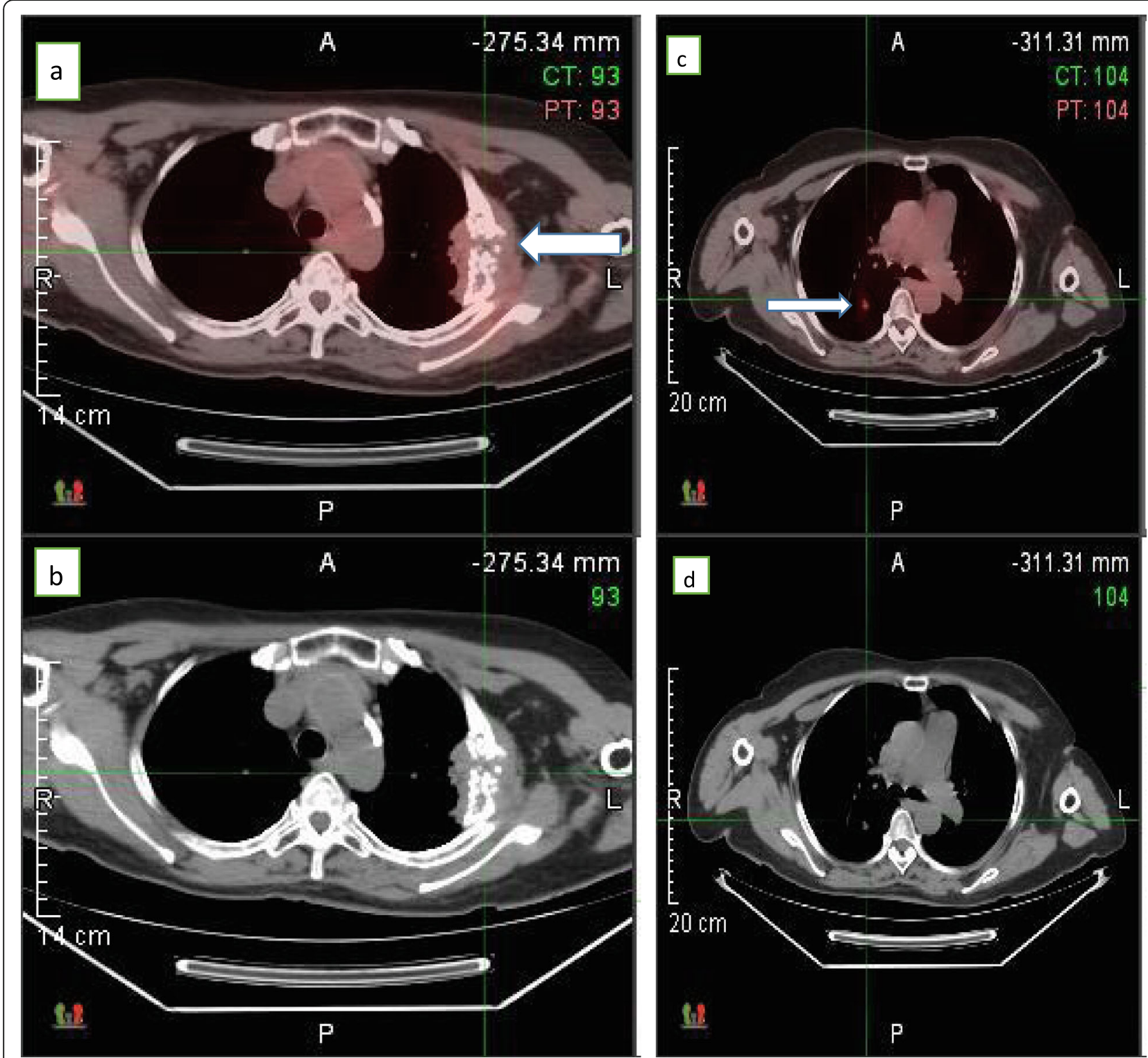

Fig. 3 (a-d) 65-year-old female with 2 years status post chemotherapy 18F-FDG PET/CT revealed stationary course of the left lateral upper lobe chest wall mass $(7.3 \mathrm{cmAP} \times 5.7 \mathrm{~cm}$ vertical distance $\times 3.5 \mathrm{~cm}$ wide) with coarse dystrophic calcification and underlying reconstituted rib cage. Lesion demonstrates low-grade FDG activity $\left(S U V_{\max }\right.$ 1.4) (arrow) (a, b). On follow-up, new $1 \mathrm{~cm}$ FDG avid right posterior lung nodule (SUV $V_{\max }$ 2.1) (arrow) consistent with neoplasm was detected (c, d)

Table 3 Group B ( $n=34)$ follow-up imaging results (correlation with final diagnosis)

\begin{tabular}{lll}
\hline & 18 F-FDG PET/CT & CECT \\
\hline Remission & 9 & 10 \\
True negative & 2 & 1 \\
False positive & & \\
Recurrent or residual & 0 & 7 \\
False negative & 23 & 16 \\
True positive & & \\
\hline
\end{tabular}

F-FDG PET/CT is a superior modality for detecting recurrent lung tumor compared with other conventional imaging modalities including chest X-ray, CT, bone scintigraphy, and MRI. The pooled sensitivity and specificity were found to be $90 \%$ and $90 \%$ respectively for $18 \mathrm{~F}$ FDG PET/CT and $78-80 \%$ respectively for conventional imaging [9].

Sawada S. et al. and Alexander J. et al. just compared 18 F-FDG PET/CT and CECT in detecting recurrent or persistence tumor during follow-up period, they concluded $97-100 \%$ sensitivity and 62- 


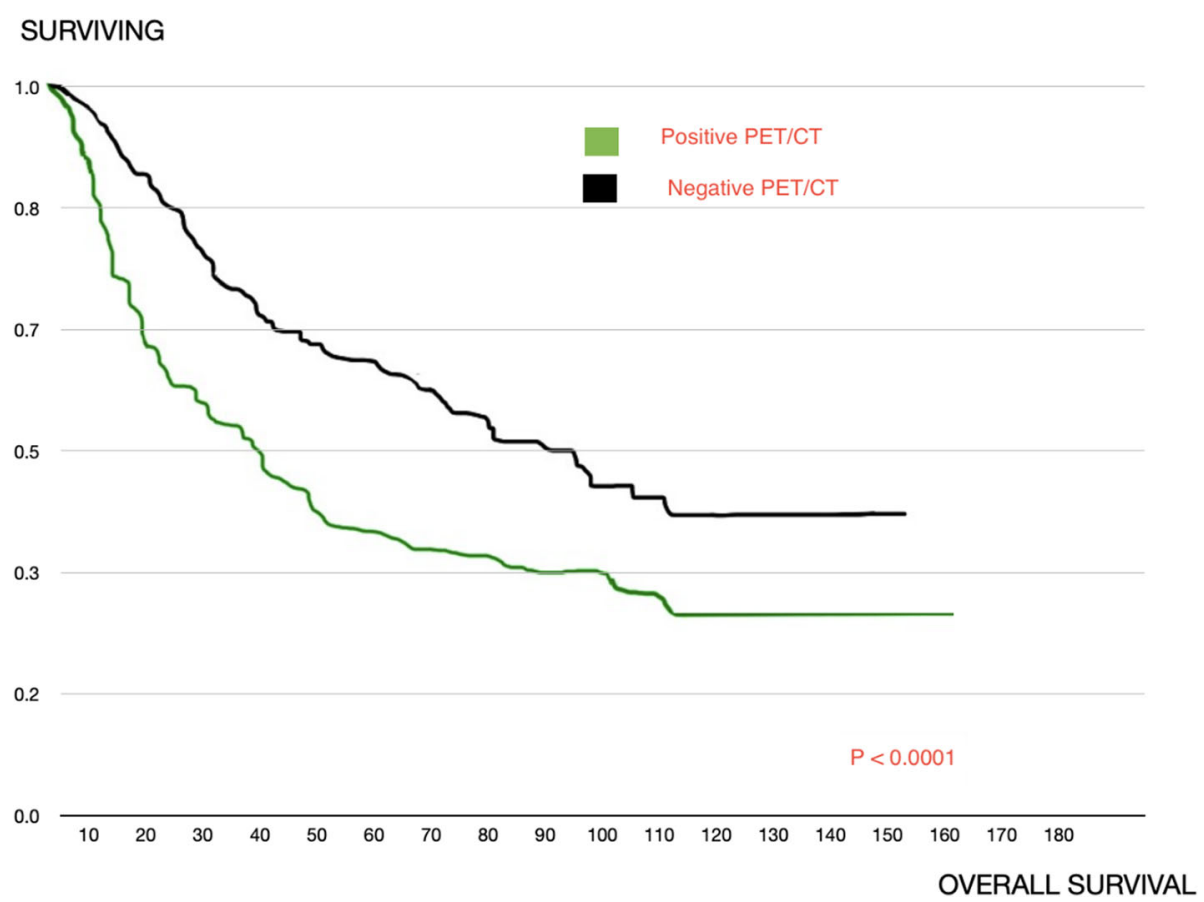

Fig. 4 Kaplan-Meier survival analysis showed significant difference of the overall survival of patients with 18F-FDG PET/CT positive and negative results for tumor

$100 \%$ specificity of 18 F-FDG PET/CT. However, they considered it for follow-up assessment when it is performed more than 6 months after primary treatment completion [10, 11].

The present study demonstrated that 18 F-FDG PET/CT positive result for tumor relapse were accompanied with decreased overall survival of patients $(P<0.0001)$ regardless of the timing for performing the scan during the follow-up or surveillance period .This agreed with Coche E. published research which found a significant negative relationship between overall survival and positive 18 F-FDG PET/CT results [12].

Barnett SA. et al. demonstrated that 18 F-FDG PET/ CT was of a significant prognostic indicator of overall survival regardless of the timing of the scan; hence, it may lead to a significant change in management of lung cancer patients [13].

In controversy, a previous study done by Ettinger DS et al. stated that $18 \mathrm{~F}-\mathrm{FDG} \mathrm{PET} / \mathrm{CT}$ is better performed when there is clinical suspicious of recurrent tumor or metastases and according to the patients clinical symptoms or laboratory examinations findings. They stated that 18 F-FDG PET/CT as routine surveillance without any clinical suspicious should be discouraged [14]. However, other authors Budak $\mathrm{E}$ et al. concluded that $18 \mathrm{~F}$ FDG PET/CT could potentially become an alternative to conventional follow-up modalities after treatment therapy [15].
Limitations for the present study were the small number of patients and the high cost of the technique which justify further research.

\section{Conclusion}

18 F-FDG PET/CT plays an important role in distinguishing post treatment changes from tumor recurrence in patients with lung cancer. Follow-up or surveillance 18 F-FDG PET/CT is of great value for overall survival of patients. However, the prognostic value and the cost effectiveness in follow-up need to be further investigated by large scale multi-center study.

\section{Abbreviations}

NSCLC: Non-small cell lung cancer; 18 F-FDG PET/CT: 18F fluorodeoxyglucose positron emission tomography/computed tomography; CECT: Contrastenhanced computed tomography; SUV: Standard uptake value; PPV: Positive predictive value; NPV: Negative predictive value

\section{Acknowledgements}

Not applicable.

\section{Author's contributions}

The single author is solely responsible to the design and implementation of the research, to the analysis of the results and to the writing of the manuscript. The author read and approved the final manuscript.

\section{Funding}

None.

\section{Availability of data and materials}

The datasets used and/or analyzed during the current study are available from the corresponding author on reasonable request. 


\section{Declarations}

\section{Ethics approval and consent to participate}

This study was approved by the Research Ethics Committee of the Faculty of Medicine at October 6 University, reference number not applicable. All patients gave written informed consent to participate in this study which was approved by the Research Ethics Committee of the Faculty of Medicine at October 6 University.

\section{Consent for publication}

All patients included in this study gave informed consent to publish the data contained within this study.

\section{Competing interests}

The author declares that she has no competing interests.

Received: 23 February 2021 Accepted: 25 April 2021

Published online: 06 May 2021

\section{References}

1. Detterbeck FC, Boffa DJ, Kim AW et al (2017) The eighth edition lung cancer stage classification. Chest 151(1):193-203. https://doi.org/10.1016/j.chest.201 6.10.010 Epub 2016 Oct 22. PMID: 27780786

2. Surveillance, Epidemiology, and End Results Program. SEER cancer statistics review, 1975-2012, lung cancer. National Cancer Institute website (2015) seer.cancer.gov/archive/csr/1975_2012/. Updated November 18, 2015. Accessed 13 Sept 2016 [Google Scholar]

3. Ettinger DS, Wood DE, Aggarwal C et al. (2019) NCCN guidelines insights: non-small cell lung cancer, Version 1.2020 J Natl Compr Canc Netw. (12):1464-1472. doi: https://doi.org/10.6004/jncen.2019.0059. PMID: 31805526

4. Osman AM, Korashi HI (2020) PET/CT implication on bronchogenic carcinoma TNM staging and follow-up using RECIST/PERCIST criteria: a comparative study with CT. Egypt J Radiol Nucl Med 51:16. https://doi.org/1 0.1186/s43055-020-0133-5

5. Veenstra CM, Vachani A, Ciunci CA et al (2016) Trends in the use of 18Ffluorodeoxyglucose PET imaging in surveillance of non-small-cell lung and colorectal cancer. J Am Coll Radiol 13:491-496 [Crossref] [Medline] [Google Scholar]

6. Jiménez-Bonilla JF, Quirce R, Martinez-Rodríguez I et al (2013) Diagnosis of recurrence and assessment of post-recurrence survival in patients with extracranial non-small cell lung cancer evaluated by 18F-FDG PET/CT. Lung Cancer 81:71-76

7. American Cancer Society (ACS). Lung cancer. ACS website(2015) www. cancer.org/cancer/lungcancer/index. Published (2015). Accessed 13 Sept 2016 [Google Scholar]

8. Sheikhbahaei S, Mena E, Yanamadala A et al (2017) The value of FDG PET/ $C T$ in treatment response assessment, follow-up, and surveillance of lung cancer. AJR Am J Roentgenol. 208(2):420-433. https://doi.org/10.2214/AJR.1 6.16532 Epub 2016 Oct 11. PMID: 27726427

9. You J, Cline K, Gu CS et al (2015) 18F-fluorodeoxyglucose positron-emission tomography-computed tomography to diagnose recurrent cancer. $\mathrm{Br} J$ Cancer 112:1737-1743. https://doi.org/10.1038/bjc.2015.151

10. Sawada S, Suehisa $H$, Ueno T et al (2016) Monitoring and management of lung cancer patients following curative-intent treatment: clinical utility of 2-deoxy-2-[fluorine-18]fluoro-d-glucose positron emission tomography/computed tomography. Lung Cancer (Auckl) 27(7):45-51. https://doi.org/10.2147/LCTT.S83644 PMID: 28210160; PMCID: PMC5310700

11. Antoniou AJ, Marcus C, Tahari AK et al (2014) Follow-up or surveillance 18FFDG PET/CT and survival outcome in lung cancer patients. J Nucl Med 55:17. https://doi.org/10.2967/jnumed.113.136770

12. Coche $\mathrm{E}$ (2016) Evaluation of lung tumor response to therapy: current and emerging techniques. Diagn Intervent Imaging. 97(10):1053-1065. https:// doi.org/10.1016/j.diii.2016.09.001 Epub 2016 Sep 29. PMID: 27693090

13. Barnett SA, Downey RJ, Zheng J et al (2016) Utility of routine PET imaging to predict response and survival after induction therapy for non-small cell lung cancer. Ann Thorac Surg 101:1052-1059 [Crossref] [Medline] [Google Scholar]
14. Ettinger DS, Wood DE, Aisner DL et al (2017) Nonsmall cell lung cancer, version 5.2017, NCCN clinical practice guidelines in oncology. J Natl Compr Canc Netw 15:504-535

15. Budak E, Çok G, Akgün A (2018) The contribution of fluorine 18F-FDG PET/ $\mathrm{CT}$ to lung cancer diagnosis, staging and treatment planning. Mol Imaging Radionucl Ther 27(2):73-80. https://doi.org/10.4274/mirt.53315 PMID: 29889029; PMCID: PMC5996604

\section{Publisher's Note}

Springer Nature remains neutral with regard to jurisdictional claims in published maps and institutional affiliations.

\section{Submit your manuscript to a SpringerOpen ${ }^{\circ}$ journal and benefit from:}

- Convenient online submission

- Rigorous peer review

- Open access: articles freely available online

- High visibility within the field

- Retaining the copyright to your article

Submit your next manuscript at $\boldsymbol{\nabla}$ springeropen.com 